\title{
The dawn of a universal language for an emerging malignancy
}

\author{
Junichi Shindoh \\ Hepatobiliary-pancreatic Surgery Division, Department of Gastroenterological Surgery, Toranomon Hospital, Tokyo, Japan \\ Correspondence to: Junichi Shindoh, MD, PhD. Hepatobiliary-pancreatic Surgery Division, Department of Gastroenterological Surgery, Toranomon \\ Hospital, 2-2-2 Toranomon, Minato-ku, Tokyo 105-8470, Japan. Email: shindou-tky@umin.ac.jp. \\ Comment on: Kamarajah SK. Evaluation of the AJCC 8th Edition Staging System for Pathologically Versus Clinically Staged Intrahepatic \\ Cholangiocarcinoma (iCCA): a Time to Revisit a Dogma? A Surveillance, Epidemiology, and End Results (SEER) Analysis. J Gastrointest Cancer \\ 2019;50:392-9.
}

Submitted Dec 11, 2018. Accepted for publication Dec 18, 2018.

doi: $10.21037 /$ cco.2018.12.02

View this article at: http://dx.doi.org/10.21037/cco.2018.12.02

With developments in diagnostic modalities and treatment options for cancers, general rules for cancer staging have repeatedly been revised with the times according to the anatomic site, biological behavior, and pathological characteristics of tumors. Compared with the other type of malignancies, hepatobiliary cancers are relatively rare and often considered as orphan diseases. However, the incidence of hepatobiliary cancers is rising, and accordingly, there is an urgent need for practical staging to guide treatment selection.

Nonetheless, staging of hepatobiliary cancers requires robust data based on high-quality surgery, detailed pathological diagnosis, and reliable follow-up, which are not always available from large datasets and registries (1). Therefore, the American Joint Committee on Cancer (AJCC) staging system is largely based on single-institution series from high-volume centers of excellence in surgery and pathology. Given such process for launching a new cancer staging manual from the table of the AJCC task force meeting, subsequent validation process using a large dataset is important to confirm the performance of the new system for dictating cancer stages.

In this issue of Chinese Clinical Oncology, Kamarajah reports a validation study for the $8^{\text {th }}$ edition of AJCC system for intrahepatic cholangiocarcinoma (ICC) using a surveillance, epidemiology, and end results (SEER) database. ICC is a rare malignancy, accounting for $10-15 \%$ of primary liver cancer. However, its incidence is clearly increasing (2) and the $7^{\text {th }}$ edition of AJCC system introduced a new staging for ICC, which were previously staged the same as hepatocellular carcinoma (3). In the $8^{\text {th }}$ edition, three major changes were made: (I) introduction of a new cutoff size of $5 \mathrm{~cm}$ for T1 category; (II) modification of the T2 category for reflecting the equivalent prognostic value of vascular invasion and tumor multifocality, and (III) elimination of tumor growth pattern from the T4 category. In the present validation study, Kamarajah found that the 8th edition is comparable to the 7 th edition and valid in stratifying patient prognosis, while the new staging system performs much better for prognostication of surgical population. These results are partly welcomed because the current version of staging system better dictates the pathological staging of cancer which determines the efficacy of surgery. However, as mentioned in discussion, these findings also suggest the need for improved accuracy of radiological imaging in "clinical" staging to guide prognosis of patient with ICC. Although surgery is an only curative option for patients with ICC, clinical staging is important especially for guiding future clinical trials looking at the efficacy of adjuvant/neoadjuvant therapies for this aggressive disease entity.

In addition to the AJCC staging system, liver cancer study group of Japan (LCSGJ) also presents a unique staging system for ICC (Table 1) (4). LCSGJ staging system is established on a nationwide survey for primary liver cancer (5). Based on the analysis of 419 patients with mass forming dominant type of ICC, optimal size cutoff was estimated as $2 \mathrm{~cm}$ and major biliary invasion (i.e., invasion of the first-order branch of the bile duct) was an independent prognostic factor showing close correlation with macroscopic periductal invasion. As such, these variables were included in the current definition of $\mathrm{T}$ 
Table 1 Liver Cancer Study Group of JAPAN staging system for intrahepatic cholangiocarcinoma 6th edition

\begin{tabular}{|c|c|}
\hline Variables & Parameter \\
\hline \multicolumn{2}{|l|}{ Criteria } \\
\hline No. of tumors & Solitary \\
\hline Size of largest tumor & $\leq 2 \mathrm{~cm}$ \\
\hline $\begin{array}{l}\text { Vascular or major biliary } \\
\text { invasion }\end{array}$ & vp0, va0, b0-b2 \\
\hline \multicolumn{2}{|l|}{ Tumor classification } \\
\hline $\mathrm{T} 1$ & All 3 criteria are fulfilled \\
\hline $\mathrm{T} 2$ & Only 2 of the 3 criteria are fulfilled \\
\hline T3 & Only 1 of the 3 criteria are fulfilled \\
\hline $\mathrm{T} 4$ & Nome of the 3 criteria are fulfilled \\
\hline \multicolumn{2}{|l|}{ Stage } \\
\hline I & T1N0M0 \\
\hline II & T2NOMO \\
\hline III & T3NOMO \\
\hline \multirow[t]{2}{*}{ IVA } & T4NOMO \\
\hline & T1-3N1M0 \\
\hline \multirow[t]{2}{*}{ IVB } & T4N1M0 \\
\hline & AnyTAnyNM1 \\
\hline
\end{tabular}

b0-b2, no biliary invasion or minor biliary invasion within second-order branch of the bile duct; vp0, no portal vein invasion; va0, no arterial invasion.

category. The difference between AJCC staging system and LCSGJ staging system might be attributable to the differences in median size of tumor and template for pathological examinations. However, we are still at the entrance of new staging system for this rare malignancy and now in the stage of gathering evidence and data for establishing a universally available staging system for ICC. Because the lack of level I evidence to support the staging proposals is the major limitation of the current staging systems, continuous international coordination toward high-quality evidence and validation will be required.

\section{Acknowledgments}

None.

\section{Footnote}

Conflicts of Interest: The author has no conflicts of interest to declare.

\section{References}

1. Chun YS, Pawlik TM, Vauthey JN. 8th Edition of the AJCC Cancer Staging Manual: Pancreas and Hepatobiliary Cancers. Ann Surg Oncol 2018;25:845-7.

2. Yao KJ, Jabbour S, Parekh N, et al. Increasing mortality in the United States from cholangiocarcinoma: an analysis of the National Center for Health Statistics Database. BMC Gastroenterol 2016;16:117.

3. Hyder O, Marques H, Pulitano C, et al. A nomogram to predict long-term survival after resection for intrahepatic cholangiocarcinoma: an Eastern and Western experience. JAMA Surg 2014;149:432-8.

4. Liver Cancer Study Group of Japan. The general rules for the clinical and pathological study of primary liver cancer. 6th edition. Tokyo: Kanehara, 2015.

5. Sakamoto Y, Kokudo N, Matsuyama Y, et al. Proposal of a new staging system for intrahepatic cholangiocarcinoma: Analysis of surgical patients from a nationwide survey of the Liver Cancer Study Group of Japan. Cancer 2016;122:61-70.
Cite this article as: Shindoh J. The dawn of a universal language for an emerging malignancy. Chin Clin Oncol 2019;8(Suppl 1):S7. doi: 10.21037/cco.2018.12.02 